\title{
STABILITY OF ISOMETRIES ON BANACH SPACES
}

\author{
JULIAN GEVIRTZ 1
}

\begin{abstract}
Let $X$ and $Y$ be Banach spaces. A mapping $f: X \rightarrow Y$ is called an $\varepsilon$-isometry if $\left|\left\|f\left(x_{0}\right)-f\left(x_{1}\right)\right\|-\left\|x_{0}-x_{1}\right\|\right| \leqslant \varepsilon$ for all $x_{0}, x_{1} \in X$. It is shown that there exist constants $A$ and $B$ such that if $f: X \rightarrow Y$ is a surjective $\varepsilon$-isometry, then $\left\|f\left(\left(x_{0}+x_{1}\right) / 2\right)-\left(f\left(x_{0}\right)+f\left(x_{1}\right)\right) / 2\right\| \leqslant A\left(\varepsilon\left\|x_{0}-x_{1}\right\|\right)^{1 / 2}+B \varepsilon$ for all $x_{0}, x_{1} \in X$. This, together with a result of Peter M. Gruber, is used to show that if $f: X \rightarrow Y$ is a surjective $\varepsilon$-isometry, then there exists a surjective isometry $I: X \rightarrow Y$ for which $\|f(x)-I(x)\| \leqslant 5 \varepsilon$, thus answering a question of Hyers and Ulam about the stability of isometries on Banach spaces.
\end{abstract}

Throughout, $X, Y$ and $Z$ denote real Banach spaces. A mapping $f: X \rightarrow Y$ is called an $\varepsilon$-isometry if $\left|\left\|f\left(x_{0}\right)-f\left(x_{1}\right)\right\|-\left\|x_{0}-x_{1}\right\|\right| \leqslant \varepsilon$ for all $x_{0}, x_{1} \in X$. Hyers and Ulam [3] formulated the stability problem for isometries, that is, the question as to whether for each pair of Banach spaces $X$ and $Y$ there exists a constant $K=$ $K(X, Y)$ such that for each surjective $\varepsilon$-isometry $f: X \rightarrow Y$ there exists an isometry $I$ : $X \rightarrow Y$ for which $\|f(x)-I(x)\| \leqslant K \varepsilon$ for all $x \in X$. This problem has been solved in a number of special cases (see [1] and [2] for a summary of such results), and Gruber [2, Theorem 1] went very far towards a general solution by showing that if $f$ : $X \rightarrow Y$ is a surjective $\varepsilon$-isometry and $I: X \rightarrow Y$ is an isometry for which $I(0)=f(0)$ and for which $\|f(x)-I(x)\| /\|x\| \rightarrow 0$ uniformly as $\|x\| \rightarrow \infty$, then $I$ is surjective and $\|f(x)-I(x)\| \leqslant 5 \varepsilon$ for all $x \in X$. In what follows we will show that such an isometry always exists so that the answer to the question of Hyers and Ulam is affirmative with $K(X, Y)=5$ for all $X$ and $Y$. We do this by establishing that:

There exist constants $A$ and $B$ such that if $f: X \rightarrow Y$ is a surjective $\varepsilon$-isometry, then

$$
\begin{aligned}
& \left\|f\left(\left(x_{0}+x_{1}\right) / 2\right)-\left(f\left(x_{0}\right)+f\left(x_{1}\right)\right) / 2\right\| \leqslant A\left(\varepsilon\left\|x_{0}-x_{1}\right\|\right)^{1 / 2}+B \varepsilon \\
& \text { for all } x_{0}, x_{1} \in X .
\end{aligned}
$$

(We show this with $A=10$ and $B=20$, but the specific values of $A$ and $B$ are of no consequence.)

To see that (1) indeed proves the existence of the isometry $I$ of Gruber's result we may assume without loss of generality that $f(0)=0$. Applying (1) with $x_{0}=2^{n+1} x$

\footnotetext{
Received by the editors December 14, 1982 and, in revised form, April 22, 1983.

1980 Mathematics Subject Classification. Primary 46B99.

Key words and phrases. $\varepsilon$-isometry, stability of isometries.

'This work was supported by a grant from the Direccion de Investigacion of the Universidad Catolica de Chile.
} 
and $x_{1}=0$ and dividing by $2^{n}$ we have

$$
\left\|2^{-n} f\left(2^{n} x\right)-2^{-n-1} f\left(2^{n+1} x\right)\right\| \leqslant 2^{-n / 2} A(2 \varepsilon\|x\|)^{1 / 2}+2^{-n} B \varepsilon .
$$

Since

$$
f(x)-2^{-n} f\left(2^{n} x\right)=\sum_{k=0}^{n-1}\left(2^{-k} f\left(2^{k} x\right)-2^{-k-1} f\left(2^{k+1} x\right)\right),
$$

the completeness of $Y$ together with (2) implies that $I(x)=\lim _{n \rightarrow \infty} 2^{-n} f\left(2^{n} x\right)$ exists for all $x \in X$ and satisfies $I(0)=0$ and

$$
\|f(x)-I(x)\| \leqslant 2(\sqrt{2}-1)^{-1} A(\varepsilon\|x\|)^{1 / 2}+2 B \varepsilon
$$

so that $\|f(x)-I(x)\| /\|x\| \rightarrow 0$ uniformly as $\|x\| \rightarrow \infty$. Since

$$
\left\|2^{-n} f\left(2^{n} x_{0}\right)-2^{-n} f\left(2^{n} x_{1}\right)\right\|-\left\|x_{0}-x_{1}\right\| \mid \leqslant \varepsilon / 2^{n},
$$

it is clear that $I$ is an isometry. We mention, in passing, that an observation of Gruber [2, Remark, p. 266] shows that (1) serves to eliminate some of his considerations. Indeed, (1) implies that $I\left(\left(x_{0}+x_{1}\right) / 2\right)=\left(I\left(x_{0}\right)+I\left(x_{1}\right)\right) / 2$ so that if we assume as above that $f(0)=0$, it follows that $I$ is linear and in turn surjective.

To facilitate the proof of (1) we introduce some terminology. If $f: X \rightarrow Y$, then any mapping $F: Y \rightarrow X$ for which

$$
\|f F(y)-y\| \leqslant \delta \quad \text { for all } y \in Y
$$

is said to be a $\delta$-inverse of $f$. Following Bourgin [1] $f$ is called $\delta$-onto if it has a $\delta$-inverse. Henceforth the term $(\delta, \varepsilon)$-isometry will refer to a $\delta$-onto $\varepsilon$-isometry. We have:

If $f: X \rightarrow Y$ is a $(\delta, \varepsilon)$-isometry and $F$ is a $\delta$-inverse of $f$, then

$F$ is a $(\delta+\varepsilon, 2 \delta+\varepsilon)$-isometry.

To see that $F$ is $(\delta+\varepsilon)$-onto we note that

$$
\|F f(x)-x\| \leqslant\|f F f(x)-f(x)\|+\varepsilon \leqslant \delta+\varepsilon
$$

by (4). To see that $F$ is a $(2 \delta+\varepsilon)$-isometry, let $y_{0}, y_{1} \in Y$. Then $\left\|f F\left(y_{i}\right)-y_{i}\right\| \leqslant \delta$ $(i=0,1)$ by $(4)$ and

$$
\left|\left\|f F\left(y_{0}\right)-f F\left(y_{1}\right)\right\|-\left\|F\left(y_{0}\right)-F\left(y_{1}\right)\right\|\right| \leqslant \varepsilon
$$

since $f$ is an $\varepsilon$-isometry. Hence

$\left|\left\|F\left(y_{0}\right)-F\left(y_{1}\right)\right\|-\left\|y_{0}-y_{1}\right\|\right| \leqslant\left|\left\|f F\left(y_{0}\right)-f F\left(y_{1}\right)\right\|-\left\|y_{0}-y_{1}\right\|\right|+\varepsilon \leqslant 2 \delta+\varepsilon$.

We shall also need:

Let $f_{1}: X \rightarrow Y$ be a $\left(\delta_{1}, \varepsilon_{1}\right)$-isometry and let $f_{2}: Y \rightarrow Z$ be a $\left(\delta_{2}, \varepsilon_{2}\right)$-isometry. Then $f_{2} f_{1}$ is a $\left(\delta_{1}+\delta_{2}+\varepsilon_{2}, \varepsilon_{1}+\varepsilon_{2}\right)$ isometry.

It is immediate that $f_{2} f_{1}$ is an $\left(\varepsilon_{1}+\varepsilon_{2}\right)$-isometry. To see the rest, let $F_{i}$ be a $\delta_{i}$-inverse of $f_{i}(i=1,2)$ and let $z \in Z$. Then

$$
\begin{aligned}
\left\|f_{2} f_{1} F_{1} F_{2}(z)-z\right\| & \leqslant\left\|f_{2} f_{1} F_{1} F_{2}(z)-f_{2} F_{2}(z)\right\|+\delta_{2} \\
& \leqslant\left\|f_{1} F_{1} F_{2}(z)-F_{2}(z)\right\|+\varepsilon_{2}+\delta_{2} \\
& \leqslant \delta_{1}+\varepsilon_{2}+\delta_{2}
\end{aligned}
$$


where we have used (4) as it applies to $f_{2}$ and $f_{1}$ and the fact that $f_{2}$ is an $\varepsilon_{2}$-isometry.

The proof of (1) which we now present is an adaptation of a proof given by Vogt [4] of a generalization of the Mazur-Ulam theorem on isometries. Let $x_{0}, x_{1} \in X$, $y_{i}=f\left(x_{i}\right)(i=0,1), p=\left(x_{0}+x_{1}\right) / 2$ and $q=\left(y_{0}+y_{1}\right) / 2$. Until the very end of the proof we shall assume that $y_{0} \neq y_{1}$. Since $f$ is a $(0, \varepsilon)$-isometry, by $(5)$ it has a 0 -inverse $F$ which is an $(\varepsilon, \varepsilon)$-isometry and for which $F\left(y_{i}\right)=x_{i}(i=0,1)$. We define sequences $\left(g_{k}\right)_{k \geqslant 0}$ and $\left(G_{k}\right)_{k \geqslant 0}$ of mappings of $Y$ into $Y$ with the following properties:

$$
\begin{gathered}
g_{k} \text { is a }\left(4^{k+1} \varepsilon, 4^{k+1} \varepsilon\right) \text {-isometry and } g_{k}\left(y_{i}\right)=y_{1-i} \quad(i=0,1), \\
G_{k} \text { is a } 4^{k+1} \varepsilon \text {-inverse of } g_{k} \text { and } G_{k}\left(y_{i}\right)=y_{1-i} \quad(i=0,1) .
\end{gathered}
$$

To begin we let $g_{0}(y)=f(2 p-F(y))$ for $y \in Y$. By $(6), g_{0}$ is a $(2 \varepsilon, 2 \varepsilon)$-isometry and it is clear that it permutes $y_{0}$ and $y_{1}$. Thus (7) holds for $k=0$. We let $G_{0}$ be any mapping which satisfies (8) for $k=0$. Next, we let $g_{1}(y)=G_{1}(y)=2 q-y$ for $y \in Y$. Obviously (7) and (8) are then satisfied for $k=1$. Finally, assuming that we have $g_{0}, \ldots, g_{n}$ and $G_{0}, \ldots, G_{n}$ which satisfy the stipulated conditions, we define $g_{n+1}=g_{n-1} g_{n} G_{n-1}$. A simple argument based on (5) and (6) shows that $g_{n+1}$ satisfies (7) with $k=n+1 . G_{n+1}$ is then taken to be any mapping satisfying (8) with $k=n+1$.

We next define a sequence $\left(a_{n}\right)_{n \geqslant 1}$ of points of $Y$ recursively by $a_{1}=q$ and $a_{n+1}=g_{n-1}\left(a_{n}\right)$ for $n \geqslant 1$. Let $d=\left\|y_{0}-y_{1}\right\| / 2$. Denoting by $B(y, r)$ the closed ball of radius $r$ and center $y$, we have that $g_{k}\left(B\left(y_{i}, r\right)\right) \subset B\left(y_{1-i}, r+4^{k+1} \varepsilon\right)$. Since $a_{1} \in B\left(y_{0}, d\right) \cap B\left(y_{1}, d\right)$ and $a_{n}=g_{n-2} g_{n-3} \cdots g_{0}\left(a_{1}\right)$, successive application of this inclusion with $k=0,1, \ldots, n-2$ yields

$$
a_{n} \in B\left(y_{0}, d+4^{n} \varepsilon\right) \cap B\left(y_{1}, d+4^{n} \varepsilon\right) \subset B\left(q, d+4^{n} \varepsilon\right) .
$$

Since the diameter of this last ball is $2\left(d+4^{n} \varepsilon\right)$ we conclude that

$$
\left\|a_{n}-a_{n-1}\right\| \leqslant 2\left(d+4^{n} \varepsilon\right) \text { for } n \geqslant 2 .
$$

We now show that for all $y \in Y$ there holds

$$
\left\|g_{n}(y)-y\right\| \geqslant 2\left\|a_{n}-y\right\|-2\left(4^{n}-1\right) \varepsilon \text { for } n \geqslant 1 .
$$

Since $g_{1}(y)=2 q-y$, this is true for $n=1$. Assuming that it is valid for a given $n \geqslant 1$, we have

$$
\begin{aligned}
\left\|g_{n+1}(y)-y\right\| & =\left\|g_{n-1} g_{n} G_{n-1}(y)-y\right\| \\
& \geqslant\left\|g_{n-1} g_{n} G_{n-1}(y)-g_{n-1} G_{n-1}(y)\right\|-4^{n} \varepsilon \\
& \geqslant\left\|g_{n} G_{n-1}(y)-G_{n-1}(y)\right\|-2 \cdot 4^{n} \varepsilon \\
& \geqslant 2\left\|a_{n}-G_{n-1}(y)\right\|-\left(4^{n+1}-2\right) \varepsilon \\
& \geqslant 2\left(\left\|g_{n-1}\left(a_{n}\right)-g_{n-1} G_{n-1}(y)\right\|-4^{n} \varepsilon\right)-\left(4^{n+1}-2\right) \varepsilon \\
& \geqslant 2\left(\left\|a_{n+1}-y\right\|-2 \cdot 4^{n} \varepsilon\right)-\left(4^{n+1}-2\right) \varepsilon \\
& =2\left\|a_{n+1}-y\right\|-2\left(4^{n+1}-1\right) \varepsilon,
\end{aligned}
$$

so that (10) holds for all $n \geqslant 1$ by induction. (Here we have used in order: the definition of $g_{n+1},(4)$ as applied to $g_{n-1}$ with $\delta=4^{n} \varepsilon$, the fact that $g_{n-1}$ is a 
$4^{n} \varepsilon$-isometry, the inductive hypothesis, the fact that $g_{n-1}$ is a $4^{n} \varepsilon$-isometry once again, and finally the definition of $a_{n+1}$ together with (4) as applied to $g_{n-1}$.) The bound (10) implies that $\left\|a_{n+1}-a_{n}\right\|=\left\|g_{n-1}\left(a_{n}\right)-a_{n}\right\| \geqslant 2\left\|a_{n}-a_{n-1}\right\|-2$. $4^{n-1} \varepsilon$, which by induction gives

$$
\left\|a_{n}-a_{n-1}\right\| \geqslant 2^{n-2}\left\|a_{2}-a_{1}\right\|-4^{n-1} \varepsilon .
$$

Together with (9) this means that, for $n \geqslant 2,\left\|a_{2}-a_{1}\right\|$ is bounded above by $2^{2-n}\left(2 d+2 \cdot 4^{n} \varepsilon+4^{n-1} \varepsilon\right)$ or, equivalently,

$$
\left\|a_{2}-a_{1}\right\| \leqslant 2\left(d 2^{-n}+18 \varepsilon 2^{n}\right) \text { for } n \geqslant 0 .
$$

We have

$$
\begin{aligned}
\left\|a_{2}-a_{1}\right\| & =\|f(2 p-F(q))-q\|=\|f(2 p-F(q))-f F(q)\| \\
& \geqslant 2\|p-F(q)\|-\varepsilon \geqslant 2(\|f(p)-f F(q)\|-\varepsilon)-\varepsilon \\
& =2\|f(p)-q\|-3 \varepsilon,
\end{aligned}
$$

so that by (11)

$$
\|f(p)-q\| \leqslant d 2^{-n}+18 \varepsilon 2^{n}+2 \varepsilon \text { for } n \geqslant 0 .
$$

For the moment we assume that $d>18 \varepsilon$ and let $t$ be such that $d 2^{-t}=18 \varepsilon 2^{t}$; that is, $t=(\log 4)^{-1} \log (d / 18 \varepsilon)>0$. If we let $n$ be the greatest integer less than or equal to $t$, the above bound for $\|f(p)-q\|$ gives

$$
\begin{aligned}
\|f(p)-q\| & \leqslant 2 d 2^{-t}+18 \varepsilon 2^{t}+2 \varepsilon=3 d 2^{-t}+2 \varepsilon \\
& =3(18 \varepsilon d)^{1 / 2}+2 \varepsilon \leqslant 10\left(\varepsilon\left\|x_{0}-x_{1}\right\|\right)^{1 / 2}+2 \varepsilon,
\end{aligned}
$$

since $\left\|x_{0}-x_{1}\right\| \geqslant\left\|y_{0}-y_{1}\right\|-\varepsilon=2 d-\varepsilon \geqslant 35 d / 18$. On the other hand, if $d \leqslant 18 \varepsilon$ (which covers the case $y_{0}=y_{1}$ that was excluded at the beginning of the proof), then $\left\|y_{0}-y_{1}\right\| \leqslant 36 \varepsilon$ and so $\left\|x_{0}-x_{1}\right\| \leqslant 37 \varepsilon$. Thus $\left\|x_{i}-p\right\| \leqslant 19 \varepsilon$ and, consequently, $\left\|y_{i}-f(p)\right\| \leqslant 20 \varepsilon(i=0,1)$. Since $q=\left(y_{0}+y_{1}\right) / 2$ we have $\|f(p)-q\| \leqslant 20 \varepsilon$. Therefore in either case there holds $\|f(p)-q\| \leqslant 10\left(\varepsilon\left\|x_{0}-x_{1}\right\|\right)^{1 / 2}+20 \varepsilon$.

\section{REFERENCES}

1. R. D. Bourgin, Approximate isometries on finite dimensional Banach spaces. Trans. Amer. Math. Soc. 207 (1975), 309-328.

2. P. M. Gruber, Stability of isometries, Trans. Amer. Math. Soc. 245 (1978), 263-277.

3. D. H. Hyers and S. M. Ulam, On approximate isometries, Bull. Amer. Math. Soc. 51 (1945), 288-292.

4. A. Vogt, Maps which preserve equality of distance, Studia Math. 45 (1973), 43-48.

Facultad de Matemáticas, Pontificia Universidad Católica de Chile, Casilla 114 - D, Santiago, Chile. 